\title{
Membrane amplitude and triaxial stress in twisted bilayer graphene deciphered using first-principles directed elasticity theory and scanning tunneling microscopy
}

\author{
M. Neek-Amal, ${ }^{1,2}$ P. Xu, ${ }^{3,4}$ D. Qii, ${ }^{5}$ P. M. Thibado, ${ }^{5}$ L. O. Nyakiti, ${ }^{6}$ V. D. Wheeler, ${ }^{7}$ R. L. Myers-Ward, ${ }^{7}$ C. R. Eddy, Jr., ${ }^{7}$ \\ D. K. Gaskill, ${ }^{7}$ and F. M. Peeters ${ }^{2}$ \\ ${ }^{1}$ Department of Physics, Shahid Rajaee Teacher Training University, Lavizan, Tehran 16785-136, Iran \\ ${ }^{2}$ Department of Physics, University of Antwerpen, Groenenborgerlaan 171, B-2020 Antwerpen, Belgium \\ ${ }^{3}$ Laboratory for Physical Sciences, College Park, MD 20740, USA \\ ${ }^{4}$ Department of Physics, University of Maryland, College Park, MD 20740, USA \\ ${ }^{5}$ Department of Physics, University of Arkansas, Fayetteville, AR 72701, USA \\ ${ }^{6}$ U.S. Naval Research Laboratory, Washington, District of Columbia 20375, USA \\ ${ }^{7}$ Departments of Marine Engineering, Material Science and Engineering, Texas A\&M University, College Station, TX 77843, USA
}

(Received 28 April 2014; revised manuscript received 3 July 2014; published 1 August 2014)

\begin{abstract}
Twisted graphene layers produce a moiré pattern (MP) structure with a predetermined wavelength for a given twist angle. However, predicting the membrane corrugation amplitude for any angle other than pure AB-stacked or AA-stacked graphene is impossible using first-principles density functional theory (DFT) due to the large supercell. Here, within elasticity theory, we define the MP structure as the minimum-energy configuration, thereby leaving the height amplitude as the only unknown parameter. The latter is determined from DFT calculations for AB- and AA-stacked bilayer graphene in order to eliminate all fitting parameters. Excellent agreement with scanning tunneling microscopy results across multiple substrates is reported as a function of twist angle.
\end{abstract}

DOI: 10.1103/PhysRevB.90.064101

PACS number(s): 73.22.Pr, 61.48.Gh, 68.37.Ef, 73.20.At

\section{INTRODUCTION}

The electronic properties of twisted stacked graphene layers have been the focus of numerous studies [1]. The periodic potential of the interacting substrate is the source of a new set of Dirac points in the energy spectrum of graphene [2]. Also, the van Hove singularity is found to shift with twist angle $[3,4]$. For large angles, the graphene layers behave like isolated sheets, while for small angles, the new Dirac cones result in two van Hove singularities [5].

Early experimental studies of multilayer twisted graphene using scanning tunneling microscopy (STM) found a moiré pattern (MP) structure [4]. Such a MP results in an additional corrugation as compared to the untwisted case. The most prominent examples have come from epitaxial graphene grown on $\mathrm{SiC}$ [6-8]. From those experimental data, a simple analytic expression for the wavelength of the superstructure was quickly discovered and provided a clear picture of the mechanism as well as the responsible twist angle. Much more difficult, however, is predicting the corrugation amplitude, and so far a simple analytic expression for this does not exist. Theoretical studies about the height deformation of the twisted bilayer graphene are difficult. This is because the large-size (e.g., up to $10 \mathrm{~nm}$ in size) MP unit cell makes $a b$ initio calculations infeasible. Only in certain limiting cases is the size of the unit cell sufficiently small that $a b$ initio calculations are possible [9]. When density functional theory (DFT) results can be obtained, they set the standard for all other approaches. Consequently, it is best to parametrize any new approach such that it agrees with the DFT results in certain limits [10-12]. Nevertheless, there exist alternative methods that show promise using classical interatomic potentials [13].

Here we present an analytical approach for the height deformation of twisted bilayer graphene without using any fitting parameters and assuming only that the experimentally observed MP structure is the minimum-energy configuration. We show that the deformation of the top graphene layer, due to the van der Waals interaction, is affected by the MP pattern. These deformations result in strain which subsequently leads to threefold symmetry in the curvature and an induced pseudomagnetic field. We also report excellent agreement with scanning tunneling microscope measurements that we acquired from various multilayer graphene samples.

\section{THE SAMPLES AND STM EXPERIMENTS}

Multiple epitaxial graphene samples grown on various miscut (i.e., nonpolar $m$-plane and $a$-plane surfaces) $n+6 \mathrm{H}$-SiC substrates (measuring $16 \times 16 \mathrm{~mm}$, Aymont Technology) were used for this study. Growth was carried out in a commercially available hot-wall Aixtron VP508 chemical vapor deposition reactor. Prior to graphene growth, both $\mathrm{SiC}$ substrates were etched in situ in a $100 \mathrm{mbar}_{2} \mathrm{H}_{2}$ ambient at either $1520{ }^{\circ} \mathrm{C}$ or $1560{ }^{\circ} \mathrm{C}$ for $50 \mathrm{~min}$. After etching, the ambient condition was switched to $\mathrm{Ar}$, followed by a temperature ramp to $1620{ }^{\circ} \mathrm{C}$. The graphene synthesis process was then conducted for $15 \mathrm{~min}$ up to 60 min under a flowing Ar environment of 20 standard liters per min at 100 mbar, with a substrate temperature still at $1620^{\circ} \mathrm{C}$. The postgrowth morphology was characterized using atomic force microscopy and the multilayer graphene coverage was confirmed using Raman spectroscopy. After these characterizations, constant-current filled-state STM images were obtained using an Omicron ultrahigh-vacuum (base pressure is $10^{-10}$ mbar), low-temperature model STM operated at room temperature. The samples were mounted with silver paint onto a flat tantalum sample plate and transferred through a load lock into the STM chamber where it was electrically grounded. STM tips were electrochemically etched from 0.25-mm-diameter polycrystalline tungsten wire via a custom double lamella method with an automatic gravity-switch cutoff. After etching, the tips were gently rinsed with distilled water, briefly dipped in a concentrated hydrofluoric acid solution to remove surface oxides, and then transferred into the 
STM chamber. Additional experimental details are provided elsewhere [14].

\section{THE MODEL}

\section{Minimum-energy configuration}

For a given twist angle $\theta$ between two graphene layers, the top sheet is attracted to the bottom sheet due to van der Waals (vdW) interaction. The zero lattice mismatch between the honeycomb lattice structures of the two graphene layers leads to an infinite moiré wavelength $L$ when the two layers have either $\mathrm{AB}$ or $\mathrm{AA}$ stacking. This is because $L=\frac{\sqrt{3} a_{0}}{2 \sin (\theta / 2)}$, where $a_{0}=1.42 \AA$, is the carbon-carbon bond length, $\theta$ is the disorientation angle with respect to $\mathrm{AB}$ stacking having $\theta=0$, and AA stacking corresponds to $\theta=\pi / 3$. In general, the commensurate rotation, where $\mathrm{B}^{\prime}$ atom from the top layer that is directly above the A atom in the bottom layer is moved by the rotation to a position formerly occupied by an atom of the same kind, can be obtained from

$$
\theta_{n}=\cos ^{-1}\left[\frac{3 n^{2}+3 n+1 / 2}{3 n^{2}+3 n+1}\right], n=0,1,2, \ldots
$$

For twist angle $\theta$ defined with respect to the $x$ axis (taken along the zigzag chain direction of graphene), we define the out-of-plane deformation of the lattice as $h(\vec{r}, \theta)$, where $\vec{r}=(x, y)$. From experiment, we know that the minimumenergy configuration for $h(\vec{r}, \theta)$ is the MP structure and, depending on the preparation method, different twist angles are possible. Furthermore, from continuum elasticity theory, the deformation of the membrane over a flat substrate is given by the solution of the following differential equation [15]:

$$
\left[k \nabla^{4}-\tau \nabla^{2}+v(\vec{r}, \theta)\right] Z(\vec{r}, \theta)=0,
$$

where $Z(\vec{r}, \theta)$ is the height of the membrane at $\vec{r}, k$ and $\tau$ correspond to the bending and stretching modulus of graphene, and $v(\vec{r}, \theta)$ depends on the vdW parameters between the two layers and is proportional to the Hamker constant. The Fourier transform (FT) of the solution of Eq. (2) must have six moiré pattern vectors $[2,16-18]$, i.e., $\vec{G}_{m}=\Re_{\phi_{m}} \vec{G}_{0}$ with $m=0,1, \ldots, 5$, where $\vec{G}_{0}=\left(1-\Re_{\theta}\right)(0,2 \kappa)$, with $\kappa=\frac{2 \pi}{3 a_{0}}$, and $\Re_{\phi_{m}}$ (and $\Re_{\theta}$ ) is the rotation matrix about the $z$ axis over an angle $\phi_{m}=\frac{2 \pi m}{6}$ (and $\theta$ ).

Therefore, for $\theta>0$, the height deformation of graphene can be written as

$$
h(\vec{r}, \theta)=h_{0}(\theta) f(\vec{r}, \theta),
$$

where the modulation function is $f(\vec{r}, \theta)=\sum_{m} e^{i \vec{G}_{m} \cdot \vec{r}}[18]$, and $h_{0}(\theta)$ should be determined using microscopic information [the zero reference height is taken to be the AB-stacking interlayer position, i.e., $Z(\vec{r}, \theta)=d_{A B}+h(\vec{r}, \theta)$, and corresponds to the minimum-energy configuration]. For a given twist angle, we can simplify the modulation function as

$$
\begin{aligned}
h= & 2 h_{0}(\theta)\left\{\cos \left[\vec{r} \cdot \vec{G}_{0}\right]+2 \cos \left[\frac{\vec{r} \cdot \vec{G}_{0}}{2}\right]\right. \\
& \left.\times \cos \left[\frac{\sqrt{3}}{2}\left|\vec{r} \times \vec{G}_{0}\right|\right]\right\} .
\end{aligned}
$$

In order to better visualize and to compare it with experimental data, we plot this function in Fig. 1 for two typical twist angles of $1.59^{\circ}$ [Fig. 1(a)] and $1.88^{\circ}$ [Fig. 1(d)]. A plethora of STM images showing various MP (not all shown) from various substrates was collected together in order to experimentally test the theory. The two items we accurately measure from the STM images are the average wavelength as well as the average amplitude of the MPs. From the wavelength measurement, we convert to twist angle using the formula mentioned earlier. For the amplitude measurements, two situations arise. When the amplitude of the MP is large, similar to the STM images shown in Figs. 1(b) and 1(e), it is easy to determine the amplitude from the height cross-section plots similar to the ones shown in Figs. 1(c) and 1(f). Here, we show two height cross sections from Figs. 1(a) and 1(d) along the solid black lines and compare them with our experimental results (symbols) from Figs. 1(b) and 1(e).

Notice that the height profile obtained from STM measurements gives us the total height, which contains contributions from both electronic and atomic corrugations [19]. The presented theory in this work addresses only the atomic corrugations. The electronic contributions depend on the used bias voltage and the STM measurements conditions. In Ref. [3], a maximum of $50 \%$ of the total height was found to be due to the atomic corrugations. However, when the amplitude is small, the STM image shows a superposition of the MP structure and the one due to the atomic electronic corrugation. The electronic corrugation due to the individual atoms is not part of the theory. For these STM images, we measure the membrane amplitude by measuring the height change from the top of the electronic corrugation at the top of the MP to the top of the electronic corrugation at the bottom of the MP. For flat graphene or graphite, this height change gives zero. Note that it is possible that the electronic corrugation of the carbon atoms at the top of the MP is slightly different when compared to the bottom of the MP; however, we expect this to be minute given the large wavelengths and small amplitudes. The full collection of experimental STM results for membrane height versus twist angle is shown in Fig. 2 as symbols. Notice the excellent agreement between theory and experiment, which supports the idea that any electronic variation is small.

\section{ENERGETIC CONSIDERATION AND STRAIN TENSOR}

In order to have a real predictive theory, we still need to calculate $h_{0}(\theta)$. In order to do so, we first write the elastic energy as given by

$$
E_{e l}=\frac{1}{2} \int\left[k\left(\nabla^{2} h\right)^{2}+\lambda \varepsilon_{\ell \ell}^{2}+2 \mu \varepsilon_{i j}^{2}\right] d \vec{r},
$$

where $k \simeq 1.1 \mathrm{eV}$ is the bending rigidity of graphene and $\lambda=3.5 \mathrm{eV} \AA^{-2}$ and $\mu=8 \mathrm{eV}^{-2}$ are the Lamé coefficients. The elements of the strain tensor can be found using $\epsilon_{\alpha \beta}=$ $\frac{1}{2}\left(\partial_{\beta} u_{\alpha}+\partial_{\alpha} u_{\beta}\right)+\frac{1}{2} \partial_{\alpha} h \partial_{\beta} h:$

$\varepsilon_{x x}=4 h_{0}^{2}\left(\vec{G}_{0} \cdot \vec{\chi}\right)^{2}, \quad \varepsilon_{y y}=4 h_{0}^{2}\left|\vec{G}_{0} \times \vec{\chi}\right|^{2}$,

$\varepsilon_{x y}=4 h_{0}^{2}\left(\vec{G}_{0} \cdot \vec{\chi}\right)\left|\vec{G}_{0} \times \vec{\chi}\right|$, 


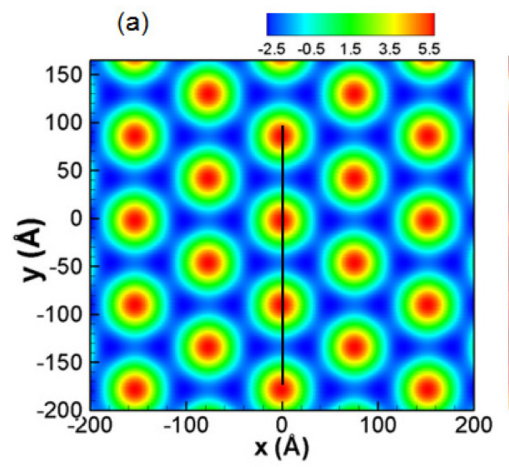

(b)

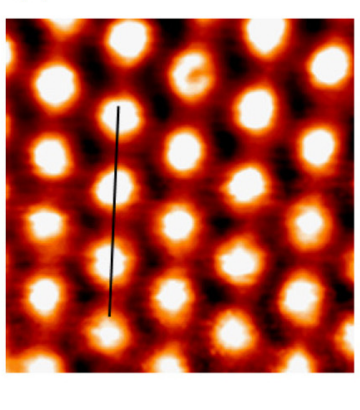

(d)

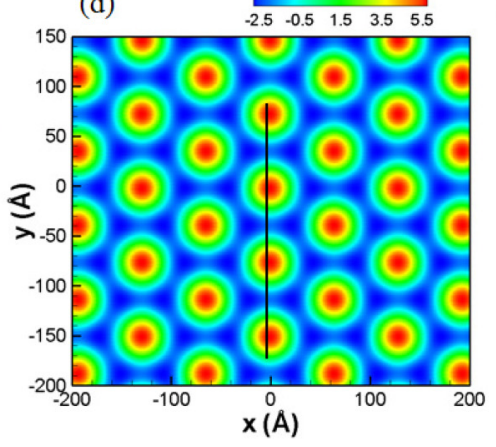

(e)

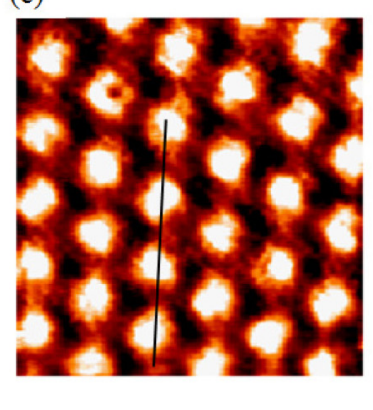

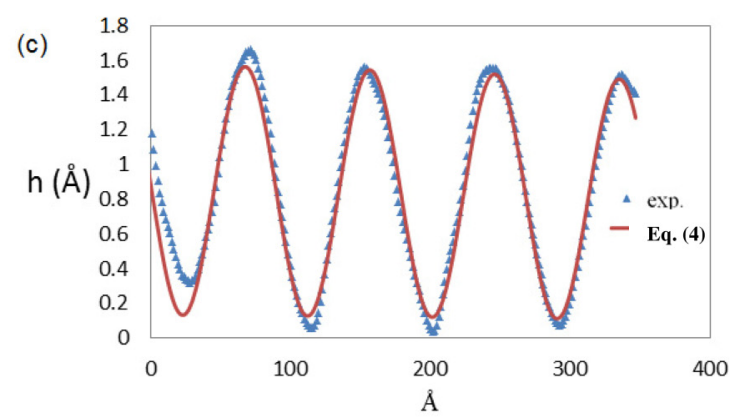

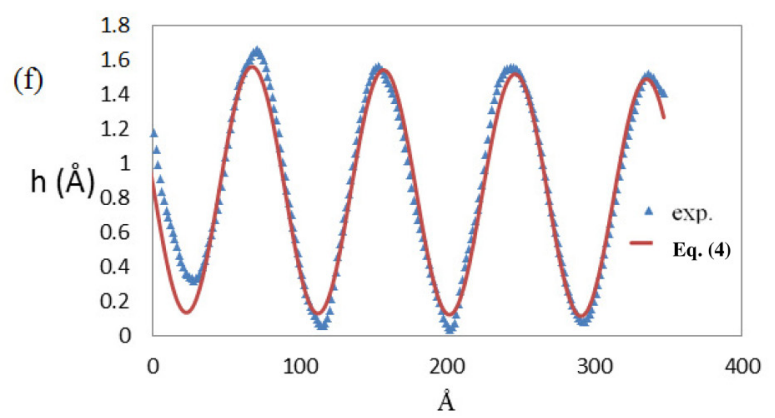

FIG. 1. (Color online) Height deformation of a graphene sheet over graphene: (a),(d) are the results from Eq. (4) and (b),(e) corresponding filled-state $(0.05 \mathrm{~V})$, constant-current (1.0 nA) STM images for twist angles (a)-(c) $\theta=1.59^{\circ}$ and (d) $-(\mathrm{f}) \theta=1.88^{\circ}$. In (c),(f), we show two cross sections along indicated solid black lines in the top figures.

$$
\begin{aligned}
\vec{\chi}= & \left\{\sin \left[\frac{\vec{r} \cdot \vec{G}_{0}}{2}\right]\left(2 \cos \left[\frac{\vec{r} \cdot \vec{G}_{0}}{2}\right]+\cos \left[\frac{\sqrt{3}}{2}\left|\vec{r} \times \vec{G}_{0}\right|\right]\right),\right. \\
& \left.\sqrt{3} \sin \left[\frac{\sqrt{3}}{2}\left|\vec{r} \times \vec{G}_{0}\right|\right] \cos \left[\frac{\vec{r} \cdot \vec{G}_{0}}{2}\right]\right\} .
\end{aligned}
$$

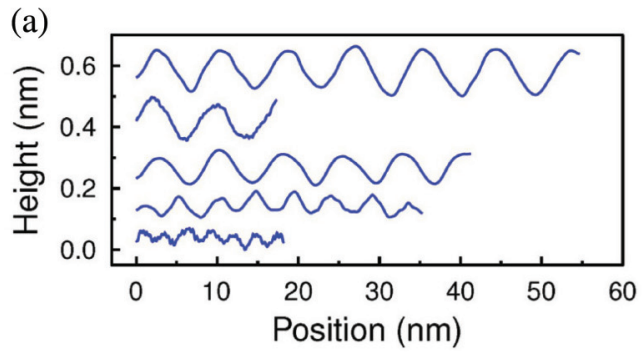

(b)

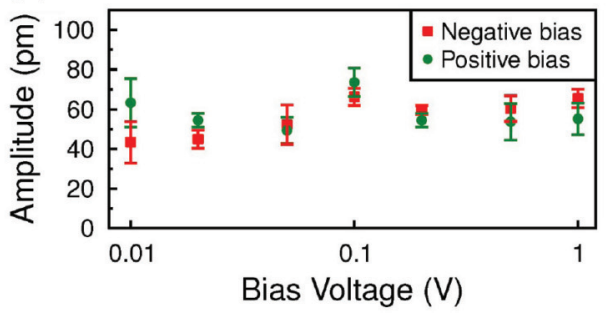

For the in-plane components (first term in parentheses), we assume that the top layer is in its minimum-energy configuration (MP structure) and the coordinate $(\vec{r})$ in our analysis is written in the deformed system, thus we do not add them to the out-of-plane components of the strain tensor (see the Appendix). Diagonalizing the strain tensor gives the

(c)

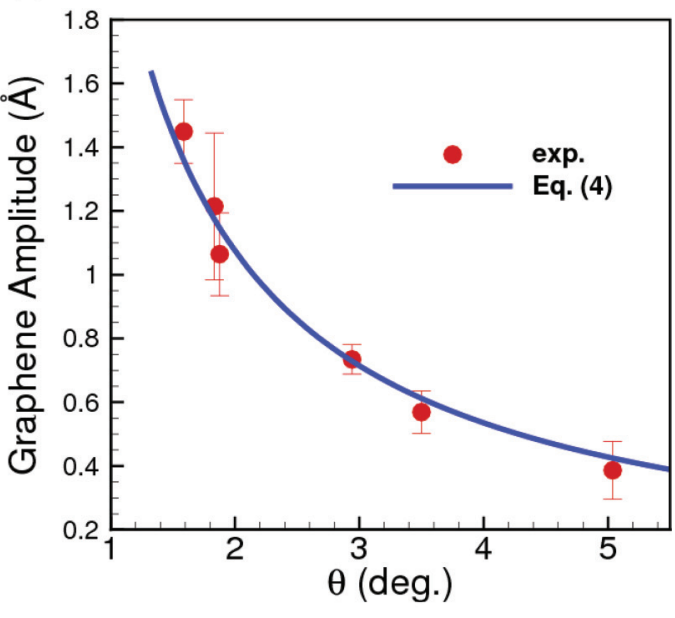

FIG. 2. (Color online) (a) Five typical constant-current height line profiles extracted from STM images acquired with a variety of tunneling set-point conditions. (b) Constant-current $(0.5 \mathrm{nA})$ STM image derived data showing the average membrane amplitude vs bias voltage set point using a semilog plot for twist angle $3.5^{\circ}$. (c) STM image derived data (symbols) showing the average membrane amplitude vs twist angle. For the STM data, we measure the wavelength $(\sim \pm 0.1 \mathrm{~nm})$ and convert to a twist angle. The solid line is the result of the presented theory, i.e., Eq. (13). 
principal axis with eigenvalues

$$
\epsilon_{ \pm}=\frac{1}{2}\left[\epsilon_{l l} \pm|\vec{A}|\right]
$$

where $\vec{A}$ is the gauge vector corresponding to the lattice deformation [20]. Surprisingly, we found that $\epsilon_{-}=0$ (since $\epsilon_{x x} \epsilon_{y y}=\epsilon_{x y}^{2}$ ) and that $\epsilon_{+}=|\vec{A}|$ has MP symmetry. Since the eigenvalue $\epsilon_{-}=0$, we conclude that the stress along the corresponding eigenvector results in no lattice deformation. These principle directions correspond to the most tensile and compression directions in graphene, i.e.,

$$
\Phi_{+}=\frac{1}{2} \tan ^{-1}\left(\frac{-A_{y}}{A_{x}}\right), \Phi_{-}=\frac{1}{2} \tan ^{-1}\left(\frac{A_{x}}{A_{y}}\right) .
$$

Solution of the integrals in Eq. (5) for a given $\theta$ can be simplified to

$$
E_{e l}=h_{0}^{2}(\theta)\left[g_{b}(\theta)+h_{0}^{2}(\theta) g_{s}(\theta)\right],
$$

where we found $g_{i}(\theta)$ by numerical integration $\left(a_{i}, b_{i}\right.$ are also fitting parameters) over the corresponding MP unit cell which has the following polynomial dependence:

$$
g_{b, s}(\theta) \cong\left(a_{b, s}+b_{b, s} \theta^{2}\right)^{2} .
$$

In our recent work [17], we presented an atomistic simulation showing that the local vdW energy stored between two layers also exhibits a moiré pattern structure. Here, using the latter idea, we write the binding energy as

$$
E_{\text {bin }}=E_{A B}-\delta E\left[1-\frac{\eta(\theta)}{\eta(0)}\right],
$$

where $E_{A B} \sim 50-60 \mathrm{meV} /$ atom is the binding energy between two graphene layers in $\mathrm{AB}$ stacking and $\delta E=E_{A B}-$ $E_{A A}(\approx 13-15 \mathrm{meV} /$ atom as found from DFT in Ref. [12]). The binding energy varies with interlayer distance as $d^{-4}$, but here we only model its variation with $\theta$ and the in-plane coordinates because we are only interested in the change in height and not in its absolute position. Notice how this parametrization incorporates the known DFT results. Note that the bright feature in all moiré patterns is where we have local AA stacking of graphene. We can understand this by realizing that in between two adjacent AA stacks, there is a low-energy AB (i.e., Bernal) stacked region. Since carbon atoms in an AA stack have higher energy as compared to the one for the $A B$ stack, we expect larger amplitude in the AA-stacked region, i.e., the AB-stacked planes are closer together as compared to the AA-stacked planes.

We introduce the function $\eta(\theta)$ in Eq. (10) based on the MP as

$$
\eta(\theta)=\int f(\vec{r}, \theta) d \vec{r},
$$

which expresses the spacial average of the modulation function over graphene.

\section{HEIGHT PROFILE AND PSEUDOMAGNETIC FIELD}

In mechanical equilibrium, the binding energy is competing with the bending energy (elastic energy), and we must have

$$
E_{e l}=E_{\text {bin }} .
$$

The solution of the latter equation results in the following dependence for $h_{0}(\theta)$ :

$$
h_{0}^{2}(\theta)=-\frac{g}{2}+\left(\frac{g^{2}}{4}+\frac{4}{3 a_{0}^{2}} \frac{\left\{E_{A B}-\delta E\left[1-\frac{\eta(\theta)}{\eta(0)}\right]\right\}}{g_{s}}\right)^{1 / 2},
$$

where $g=g_{b} / g_{s}$. Because $g_{b} / g_{s} \ll 1$, we can approximate $h_{0}$ as

$$
h_{0} \cong\left(\frac{4}{3 a_{0}^{2}} \frac{\left\{E_{A B}-\delta E\left[1-\frac{\eta(\theta)}{\eta(0)}\right]\right\}}{\left(a_{s}+b_{s} \theta^{2}\right)^{4}}\right)^{1 / 4} \sim \theta^{-1},
$$

for $\theta>1^{\circ}$. Notice that the difference between the maximum and the minimum of $h$ in Eq. (4) is given by $\Delta h=8 h_{0}$. $\left(E_{\text {bin }}-\right.$ $\left.E_{A B}\right) /(-\delta E)$ approaches 1 when $\theta \rightarrow \pi / 3$. Our prediction resulting from Eq. (13) for the overall height of the membrane (i.e., $8 h_{0}$ ) is shown versus twist angle in Fig. 2 and compared with our experimental results.

We collected a variety of STM images of various multilayer graphene moiré patterns (not all are shown) from $a$-plane and $m$-plane $\mathrm{SiC}$ substrates grown under similar conditions. Five typical line profiles extracted from these STM images and having varying wavelength and amplitude are shown in Fig. 2(a). The line profiles are ordered from top to bottom based on decreasing amplitude. Notice that the lowest line profile has, superimposed on it, an even smaller amplitude and higher frequency signal. This is the electronic corrugation of the carbon atoms, and it is worth pointing out how small the electronic amplitude is when compared to the membrane corrugation. The membrane corrugation persists when imaging the moiré pattern through a range of normal bias voltage settings $( \pm 0.05$ to $\pm 1.00 \mathrm{~V})$ and tunneling current set points ( 0.05 to $1.00 \mathrm{nA})$. For example, when a moiré pattern with a wavelength of $4 \mathrm{~nm}$ is repeatedly imaged while incrementally altering the bias voltage from \pm 0.01 to $\pm 1.0 \mathrm{~V}$ with a tunneling current set point of $0.5 \mathrm{nA}$, we see only a small amplitude variation, as shown in a semilog plot of Fig. 2(b). Within the error bars, the membrane amplitude is relatively unchanged.

A plot showing the membrane amplitude as a function of twist angle is shown in Fig. 2(c). Even though it is possible that the electronic amplitude is slightly different at the crest of the membrane compared to the trough of the membrane, we believe this is within the error bars of our results. Also, unlike the image contract inversion STM data acquired from single-crystal metal surfaces [19], for twisted graphene on graphene/SiC, we do not see any significant height changes in the moiré pattern as we vary the STM tunneling condition.

It is also worthwhile to mention that the low-energy electronics of the deformed graphene can be obtained from the modified Dirac equation due to the modified hopping parameters from the tight-binding model which are now a function of the atomic positions $t(\mathbf{r})$ [21]. The Dirac Hamiltonian in the effective mass approximation in the presence of lattice deformation (here, out-of-plane deformation) introduces strain, which induces an effective gauge field $\vec{A}=$ $\frac{2 \beta_{0} \hbar}{3 a_{0} e}\left(\varepsilon_{x x}-\varepsilon_{y y},-2 \varepsilon_{x y}\right)$ where $\beta_{0}(\sim 2-3)$ is a constant [20]. Using the strain tensor components, we found an analytical 

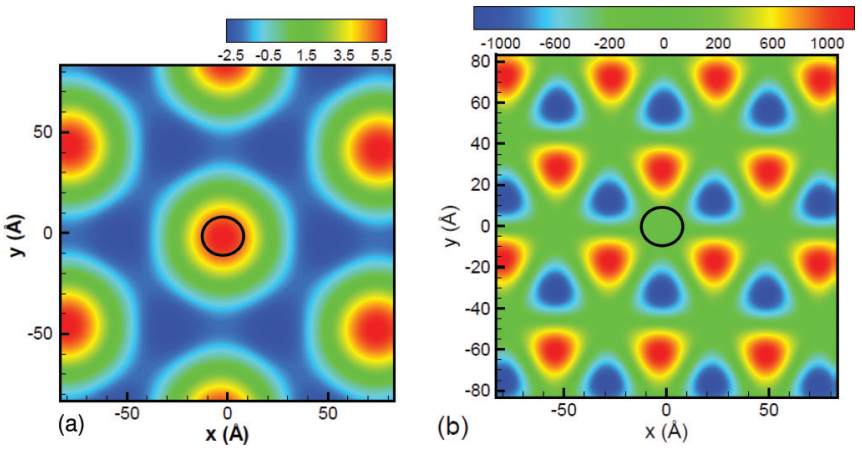

FIG. 3. (Color online) Height deformation $\left(h / h_{0}\right)$ of graphene over a graphene sheet, i.e., Eq. (4), for (a) twist angle $\theta=1.59^{\circ}$ and (b) corresponding pseudomagnetic field in units of $h_{0}^{2}$, obtained from Eq. (15). The circle in (a) and (b) indicates the regions with extreme height and zero magnetic field, respectively.

expression for $B$ as function of $\theta$,

$$
B=\frac{2 \beta_{0} \hbar}{3 a_{0} e}\left(\varepsilon_{x x, x}-\varepsilon_{y y, x}+2 \varepsilon_{x y, y}\right) .
$$

We plot the height deformation (h) in units of $h_{0}$ in Fig. 3(a) and the corresponding pseudomagnetic field in units of $h_{0}^{2}$ for $\theta=1.59^{\circ}$ in Fig. 3(b). The pseudomagnetic field has threefold symmetry and it is surprising that inside each MP unit cell, the field vanishes at the position of the extrema in the height deformation (see the circles in Fig. 3). It is also worthwhile to mention that our study realizes in a natural way the proposal for triaxial stress creation in graphene proposed by Guinea et al. [22] by using twisted graphene sheets.

\section{SUMMARY}

In summary, we presented a theory for the out-of-plane deformation of a twisted graphene sheet due to the vdW interaction with a graphene substrate. By defining the MP structure for the out-of-plane deformation as the minimum- energy configuration, we derive an analytic solution without any fitting parameters. We found excellent agreement for the height variation with our STM data for different twist angles.

\section{ACKNOWLEDGMENTS}

This work was supported by the Flemish Science Foundation (FWO-Vl) and the Methusalem Foundation of the Flemish Government. M.N.-A. was supported by the EU-Marie Curie IIF postdoctoral Fellowship No. 299855. P.M.T. is thankful for the financial support of the Office of Naval Research under Grant No. N00014-10-1-0181 and the National Science Foundation under Grant No. DMR-0855358. L.O.N. acknowledges the support of the American Society for Engineering Education and Naval Research Laboratory Postdoctoral Fellow Program. Work at the US Naval Research Laboratory is supported by the Office of Naval Research.

\section{APPENDIX}

The in-plane displacement vector for micron-size graphene flake can be written as

$$
\vec{U}=\sum_{m, n} \vec{u}\left[\vec{r}+\vec{T}_{m, n}(\theta)\right],
$$

where the summation is taken over all MP unit cells and, inside each MP unit cell (see circles in Fig. 3), one can write $\vec{u}(\vec{r}, \theta)=$ $C(\theta)\left(2 x y, x^{2}-y^{2}\right)$ as the in-plane components of the strain tensor, $\vec{T}_{m, n}(\theta)$ is the translation vector of the MP lattice, and $C$ is a twist-angle-dependent variable which determines the strength of the in-plane strain. The corresponding in-plane strain elements written for each MP unit cell are given by

$$
\varepsilon_{x x}=2 C y, \varepsilon_{y y}=-2 C y, \varepsilon_{x y}=2 C x,
$$

and the corresponding pseudomagnetic field is a function of twist angle but independent of position, i.e., $\frac{16 C \beta_{0} \hbar}{3 a_{0} e}$. The corresponding principal axes are independent of twist angle, i.e., the most tensile and compression directions in graphene are $\frac{1}{2} \tan ^{-1}\left(\frac{-x}{y}\right)$ and $\frac{1}{2} \tan ^{-1}\left(\frac{y}{x}\right)$, respectively, e.g., along the $y=x$ line, two angles are $\pm \pi / 8$.
[1] J. M. B. Lopes dos Santos, N. M. R. Peres, and A. H. Castro Neto, Phys. Rev. Lett. 99, 256802 (2007); Wen-Yu He, Zhao-Dong Chu, and Lin He, ibid. 111, 066803 (2013); E. Suárez Morell, M. Pacheco, L. Chico, and L. Brey, Phys. Rev. B 87, 125414 (2013).

[2] K. Watanabe, T. Taniguchi, P. Jarillo-Herrero, P. Jacquod, and B. J. LeRoy, Nat. Phys. 8, 382 (2012).

[3] I. Brihuega, P. Mallet, H. González-Herrero, G. Trambly de Laissardière, M. M. Ugeda, L. Magaud, J. M. Gómez-Rodríguez, F. Ynduráin, and J.-Y. Veuillen, Phys. Rev. Lett. 109, 196802 (2012).

[4] Eva Y. Andrei, G. Li, and X. Du, Rep. Prog. Phys. 75, 056501 (2012).

[5] Zhao-Dong Chu, Wen-Yu He, and Lin He, Phys. Rev. B 87, 155419 (2013); Wei Yan, Mengxi Liu, Rui-Fen Dou, Lan Meng, Lei Feng, Zhao-Dong Chu, Yanfeng Zhang, Zhongfan Liu, Jia-Cai Nie, and Lin He, Phys. Rev. Lett. 109, 126801 (2012).
[6] J. Hass, F. Varchon, J. E. Millán-Otoya, M. Sprinkle, N. Sharma, W. A. de Heer, C. Berger, P. N. First, L. Magaud, and E. H. Conrad, Phys. Rev. Lett. 100, 125504 (2008).

[7] X. Li, W. Cai, Jinho An, S. Kim, J. Nah, D. Yang, R. Piner, A. Velamakanni, I. Jung, E. Tutuc, S. K. Banerjee, L. Colombo, and R. S. Ruoff, Science 324, 1312 (2009).

[8] M. Ostler, I. Deretzis, S. Mammadov, F. Giannazzo, G. Nicotra, C. Spinella, Th. Seyller, and A. La Magna, Phys. Rev. B. 88, 085408 (2013).

[9] G. Giovannetti, P. A. Khomyakov, G. Brocks, P. J. Kelly, and J. van den Brink, Phys. Rev. B 76, 073103 (2007).

[10] L. Spanu, Sandro Sorella, and Giulia Galli, Phys. Rev. Lett. 103, 196401 (2009).

[11] A. K. Geim and I. V. Grigorieva, Nature (London) 499, 419 (2013).

[12] X. Chen, F. Tian, C. Persson, W. Duan, and N.-x. Chen, Sci. Rep. 3, 3046 (2013). 
[13] Irina V. Lebedeva, Andrey A. Knizhnik, Andrey M. Popov, Yurii E. Lozovikda, and Boris V. Potapkin, Phys. Chem. Chem. Phys. 13, 5687 (2011).

[14] P. Xu, M. L. Ackerman, S. D. Barber, J. K. Schoelz, P. M. Thibado, V. D. Wheeler, L. O. Nyakiti, R. L. Myers-Ward, C. R. Eddy, Jr., and D. K. Gaskill, Surf. Sci. 617, 113 (2013).

[15] M. Neek-Amal and F. M. Peeters, Phys. Rev. B 85, 195445 (2012).

[16] S. Tang, H. Wang, Y. Zhang, A. Li, H. Xie, X. Liu, L. Liu, T. Li, F. Huang, X. Xie, and M. Jiang, Sci. Rep. 3, 2666 (2013).

[17] M. Neek-Amal and F. M. Peeters, Appl. Phys. Lett. 104, 041909 (2014).
[18] J. R. Wallbank, A. A. Patel, M. Mucha-Kruczyński, A. K. Geim, and V. I. Fal'ko, Phys. Rev. B 87, 245408 (2013).

[19] S. Heinze, S. Blügel, R. Pascal, M. Bode, and R. Wiesendanger, Phys. Rev. B 58, 16432 (1998).

[20] A. H. Castro Neto, F. Guinea, N. M. R. Peres, K. S. Novoselov, and A. K. Geim, Rev. Mod. Phys. 81, 109 (2009).

[21] M. Neek-Amal, L. Covaci, Kh. Shakouri, and F. M. Peeters, Phys. Rev. B 88, 115428 (2013); M. Neek-Amal, L. Covaci, and F. M. Peeters, ibid. 86, 041405 (2012); M. Neek-Amal and F. M. Peeters, ibid. 85, 195446 (2012).

[22] F. Guinea, M. I. Katsnelson, and A. K. Geim, Nat. Phys. 2, 31 (2010). 